\title{
Coalgebraic Epistemic Update Without Change of Model ${ }^{\star}$
}

\author{
Corina Cîrstea and Mehrnoosh Sadrzadeh \\ School of Electronics and Computer Science, University of Southampton \\ cc2, ms6@ecs. soton.ac.uk
}

\begin{abstract}
We present a coalgebraic semantics for reasoning about information update in multi-agent systems. The novelty is that we have one structure for both states and actions and thus our models do not involve the "change-of-model" phenomena that arise when using Kripke models. However, we prove that the usual models can be constructed from ours by categorical adjunction. The generality and abstraction of our coalgebraic model turns out to be extremely useful in proving preservation properties of update. In particular, we prove that positive knowledge is preserved and acquired as a result of epistemic update. We also prove common and nested knowledge properties of epistemic updates induced by specific epistemic actions such as public and private announcements, lying, and in particular unsafe actions of security protocols. Our model directly gives rise to a coalgebraic logic with both dynamic and epistemic modalities. We prove a soundness and completeness result for this logic, and illustrate the applicability of the logic by deriving knowledge properties of a simple security protocol.
\end{abstract}

\section{Introduction}

Modelling interactive multi-agent systems has a wide range of applications, e.g. in Artificial Intelligence, computer security and e-commerce. In such systems agents communicate and as a result their knowledge gets updated, and therefore one has to model the epistemics and dynamics of the system. The Kripke and algebraic models of these settings have been presented in 9|10/3/2|14. The Kripke models have the advantage of being intuitive and concrete, while the algebraic setting benefits from high level features that result from mathematical abstraction.

In this paper we develop a coalgebraic semantics for dynamic epistemic systems, which combines the advantages of both the Kripke and the algebraic setting. Our model reasons about such systems in a uniform way, by treating both actions and agents as state transformers. Thus, we have only one structure that captures both dynamics and epistemics. This is contrary to the models of e.g. [10]3]1 that require subsequent "changes" to the epistemic structure to

\footnotetext{
* Research supported by EPSRC grant EP/D000033/1. 
model the dynamics. By "change" we mean either the update product between an epistemic Kripke structure and an action Kripke structure 3, or the update functor on the category of epistemic coalgebras 1011. In either case the epistemic structure is taken to be primitive and the dynamics is captured by operations on it. This brings us to the other novelty of our approach: we start our modelling task by fixing the epistemic actions, and then define the epistemic states based on these actions and on the agents participating in them. Again, this is contrary to the models of 10/31], which involve first fixing the epistemic states and then defining all possible epistemic actions on these states. Although at first sight our approach seems very different from the approach of [10|3|1, the two are strongly connected. In the main theorem of our paper we show how to construct from our models the models of [1] and vice versa, and prove that these constructions form a categorical adjunction 1 .

Our approach has all the advantages of the approach in 311, for instance it benefits from a general updating schema and it reflects the epistemic structure of actions. Moreover, our approach does not have the usual weaknesses, for example operations on actions are a natural part of our models, e.g. sequential composition is simply unfolding the coalgebra maps twice and does not need to be defined separately. The generality and abstraction of our coalgebraic models turns out to be extremely useful in proving preservation properties of update. In particular, we prove that positive knowledge is preserved and acquired as a result of epistemic update. We also prove common and nested knowledge properties of epistemic updates induced by specific epistemic actions such as public and private announcements, lying, and in particular unsafe actions of security protocols. Finally, our model directly gives rise to a coalgebraic logic with both epistemic and dynamic modalities. This, for instance, cannot be done for the models of 101]. We prove a soundness and completeness result for the resulting logic. As an example of application, we derive the authentication properties of a security protocol.

An extended version of this paper is available electronically [8]. There we illustrate the applicability of our models to general scenarios involving both positive and negative knowledge, by presenting a new coalgebraic proof of the muddy children puzzle and a version of it with cheating children. Our proofs are based on restrictive recursion rather than the usual induction.

\section{Coalgebraic Semantics for Actions and Agents}

We consider coalgebras of the following signature functor $T:$ Set $\rightarrow$ Set

$$
T X=\mathcal{P}_{\kappa}(X)^{A g} \times(1+X)^{A c} \times \mathcal{P}(A t)
$$

where $\kappa$ is a regular cardinal. A coalgebra map for the above functor is thus a triple $\gamma=\langle a p$, up, val $\rangle: S \rightarrow \mathcal{P}_{\kappa}(S)^{A g} \times(1+S)^{A c} \times \mathcal{P}(A t)$. The valuation map val

\footnotetext{
${ }^{1}$ As noted by one of our referees, our model might share ideas with the recent epistemic temporal models of $[4$.
} 
assigns to each state the facts that are true in that state. The (nondeterministic) appearance of states in $S$ to agents in $A g$ is modelled by the function $a p: S \rightarrow$ $\mathcal{P}(S)^{A g}$, whereas the (deterministic) effect of actions in $A c$ on states in $S$ is modelled by a function $u p: S \rightarrow(1+S)^{A c}$. Thus, up $(s)(a)$ stands for the effect of the action $a$ on the state $s$, or the update of $s$ by $a$. If this effect is the unique element $*$ of 1 , that is, if $u p(s)(a)=* 2$, we say that action $a$ does not apply in state $s$; this should be the case, for instance, when $a$ is the announcement of a fact that does not belong to $\mathrm{val}(\mathrm{s})$. The choice of functor $T$ automatically yields notions of $T$-bisimulation and $T$-bisimilarity for $T$-coalgebras (see e.g. [16]).

\subsection{Restrictions to the Coalgebras}

We are interested in using $T$-coalgebras to model the effect of communication actions on the information state or knowledge of agents. Examples of such actions are public or secret announcements, and message passing actions in a multiagent system. We want to model the effect of updates with such actions on the appearances of states to the agents and on the valuations of states. In order to limit the behaviour of our systems to the effect of these actions, we require that the coalgebra maps satisfy some additional conditions, detailed in the following.

The communication actions that we model are epistemic, that is, they only affect the information states of agents, while leaving the facts of the world unchanged. Our first restriction, called preservation of facts, reflects this point:

$$
\operatorname{val}(u p(s)(a))=\operatorname{val}(s) \quad \text { whenever } \quad u p(s)(a) \neq *
$$

It says that, if applicable to a state, an action does not change the valuation of that state. So the valuation of the effect of the action is the same as the valuation of the state before the action. We need this restriction to prove the preservation results in Section 2.2. In a more general approach, one can divide the set of actions into two subsets, namely information-changing actions and fact-changing actions, and only require this restriction for actions of the first type.

Our second restriction concerns the appearance of an update to each agent involved in the corresponding action. For applicable updates $u p(s)(a) \neq *$, this will be related to the update of each of the agent's appearances $t \in a p(s)(A)$ with a finite subset of actions $A c_{a, A} \subseteq A c$, as follows

$$
\operatorname{ap}(u p(s)(a))(A)=\left\{u p(t)\left(a^{\prime}\right) \mid t \in \operatorname{ap}(s)(A), a^{\prime} \in A c_{a, A}, u p(t)\left(a^{\prime}\right) \neq *\right\}
$$

where the actions $A c_{a, A}$ depend both on the action $a$ and on agent $A$ 's involvement in it, and are intended to capture agent $A$ 's appearance of the action $a$. This relation says that if an action $a$ applies to a state $s$, then the appearance of its effect to an agent $A$ is the same as the effect of one of the actions in $A c_{a, A}$

\footnotetext{
${ }^{2}$ Here and in what follows, we assume that $1 \cap S=\emptyset$. Under this assumption, and to simplify the notation, we regard elements of the set $1+S$ as being either $*$ or elements of $S$; that is, we make implicit the isomorphism between $1+S$ and $1 \cup S$.
} 
on one of the appearances to $A$ of the original state $s$. The case when $A c_{a, A}$ is a singleton $\left\{a^{\prime}\right\}$ corresponds to a deterministic view of $A$ about the real action $a$ (with $A$ thinking that $a^{\prime}$ is happening when in fact $a$ is happening), whereas any non-singleton set $A c_{a, A}$ captures $A$ 's uncertainty about the action taking place. We refer to the collection of all instances of this restriction (one for each action in $A c$ ) as rationality.

The content of an epistemic action, as its name suggests, describes the information that is being transmitted as a result of the action taking place. We use the following syntax to denote specific contents:

$$
\mu:=p\left|\square_{A} \mu\right| \mathrm{tt}|\neg \mu| \bigwedge_{i \in \mathbb{I}} \mu_{i}
$$

with $p \in A t$ and $\mathbb{I}$ an arbitrary set. That is, the content of an action can be a fact, the knowledge or belief of some other content by an agent the true proposition, the negation of a content, or a potentially infinite conjunction of contents 5 . In particular, the content can involve nested knowledge, as in $\square_{A} \square_{B} p$. We do not allow contents to refer to (the effect of) actions, as in $[q]-$; this avoids a circularity between requiring each action to have a content and allowing contents to depend on actions. Contents whose only occurrences of the negation operator immediately precede a fact are called positive contents, otherwise they are referred to as negative contents.

From now on, we assume that each action $a \in A$ has a content $\mu_{a}$ associated to it. Then, $a$ should be applicable precisely to those states where its content $\mu_{a}$ is satisfied. This is encoded as a further restriction on $T$-coalgebras, referred to as the content restriction:

$$
u p(s)(a) \neq * \quad \text { iff } \quad s \models \mu_{a}
$$

where the relation $\models$ between states and contents of actions is defined by structural induction on contents:

$$
\begin{aligned}
& -s \models p \quad \text { iff } \quad p \in \operatorname{val}(s) \\
& -s \models \square_{A} \mu \text { iff } \quad t \models \mu \text { for all } t \in \operatorname{ap}(s)(A)
\end{aligned}
$$

and the usual clauses for the true proposition, negation and conjunction.

Definition 1. An appearance-update coalgebra is a T-coalgebra additionally satisfying the preservation of facts, content, and rationality restrictions. We denote the set of all of these restrictions by $\mathcal{R}$.

\footnotetext{
${ }^{3}$ We emphasise that this is just a syntax for expressing our second restriction on the content $\mathrm{s}$ of actions. The logic will be presented in section 5

${ }^{4}$ Similarly to 3 and as a result of accommodating misinformation actions, our knowledge $\square_{A}$ is not necessarily truthful. Indeed, one can also think of $\square_{A}$ as belief in contexts where no wrong knowledge is allowed.

${ }^{5}$ The infinite contents are just a technicality that is needed later in order to establish a connection to the model of 1 .
} 


\subsection{Preservation and Acquisition of Knowledge}

An important consequence of the restrictions in $\mathcal{R}$ is the so-called preservation of positive contents by updates, made formal in the next result.

Proposition 1. Let $(S,\langle a p, u p, v a l\rangle)$ be an appearance-update coalgebra. Then for all positive contents $\mu$, all states $s \in S$, and all actions $a \in A c$ such that up $(s)(a) \neq *$, we have

$$
s \models \mu \quad \Longrightarrow \quad u p(s)(a) \models \mu
$$

Proof. The statement is proved by induction on $\mu$. If $\mu$ is a fact or the negation of a fact, the conclusion follows directly from the preservation of facts. Now suppose that $s \models \mu^{\prime}$ implies $u p(s)(a) \models \mu^{\prime}$ for all states $s \in S$ and applicable actions $a \in A c$. Also, let $s \in S$ and $A \in A g$ be such that $s \models \square_{A} \mu^{\prime}$. To show that $u p(s)(a) \models \square_{A} \mu^{\prime}$ for any applicable action $a$, we use the rationality restriction to reduce $\operatorname{ap}(u p(s)(a))(A)$ to $\left\{u p(t)\left(a^{\prime}\right) \mid t \in a p(s)(A), a^{\prime} \in A c_{a, A}, u p(t)\left(a^{\prime}\right) \neq *\right\}$. Thus, we must show that $u p(t)\left(a^{\prime}\right) \models \mu^{\prime}$ whenever $t \in a p(s)(A)$ and $a^{\prime} \in A c_{a, A}$ are such that $u p(t)\left(a^{\prime}\right) \neq *$. But this follows from the induction hypothesis and the assumption that $s \models \square_{A} \mu^{\prime}$. The cases when $\mu$ is the true proposition or a conjunction of contents are trivial.

The above result does not hold for negative contents. That is, there exists an appearance-update coalgebra $(S,\langle a p, u p, v a l\rangle)$, a state $s \in S$ with an applicable action $a \in A c$ and a negative content $\mu$ such that $s=\mu$ but $u p(s)(a) \models \neg \mu$. For an example of such a situation, which gives rise to the epistemic puzzle of muddy children, see [8]. It is also not possible to generalise the above result to an exclusive one for positive contents. In particular, any appearance-update coalgebra that contains in its set of actions a neutral action $\tau$ with $\mu_{\tau}=t t$ and $A c_{\tau, A}=\{\tau\}$ for all $A \in A g$ is such an example. To see why, we refer the reader to the next section where we prove that such an action preserves all contents.

Another consequence of the restrictions in $\mathcal{R}$ is the following acquisition of knowledge after updates:

Proposition 2. Let $(S,\langle a p, u p, v a l\rangle)$ be an appearance-update coalgebra. Then for all agents $A \in A g$, all states $s \in S$, and all applicable actions $a \in A c$ with positive contents $\mu_{a^{\prime}}$ for all $a^{\prime} \in A c_{a, A}$, we have

$$
u p(s)(a) \models \square_{A} \bigvee_{a^{\prime} \in A c_{a, A}} \mu_{a^{\prime}}
$$

Proof. Let $s \in S$ and $a \in A c$ be such that $u p(s)(a) \neq *$. We must show that for $A \in A g$ we have $s^{\prime} \models \bigvee_{a^{\prime} \in A c_{a, A}} \mu_{a^{\prime}}$ for all $s^{\prime} \in \operatorname{ap}(u p(s)(a))(A)$. By the rationality restriction on $\operatorname{ap}(u p(s)(a))(A)$, we must show that $u p(t)\left(a^{\prime}\right) \models \bigvee_{a^{\prime} \in A c_{a, A}} \mu_{a^{\prime}}$ whenever $t \in a p(s)(A)$ and $a^{\prime} \in A c_{a, A}$ are such that $u p(t)\left(a^{\prime}\right) \neq *$. By the content restriction, the positivity of $\mu_{a^{\prime}}$ and the preservation result we obtain $u p(t)\left(a^{\prime}\right) \models \mu_{a^{\prime}}$, which implies $u p(t)\left(a^{\prime}\right) \models \bigvee_{a^{\prime} \in A c_{a, A}} \mu_{a^{\prime}}$.

The known preservation results in the literature are special cases of our general results. For instance, it has been shown in $[3]$ that contents that do not contain the epistemic modality are preserved under any update. 


\section{Epistemic Actions}

In this section, we present epistemic actions, describe their contents and appearances, and prove their knowledge acquisition effects on agent:6.

Skip. This is the action $\tau$ in which nothing happens. We have $\mu_{\tau}=$ tt and $A c_{\tau, A}=\{\tau\}$ for all $A \in A g$. This particular choice of $\mu_{\tau}$ and $A c_{\tau, A}$ is sufficient to guarantee that, in any appearance-update coalgebra, the skip action does not affect the epistemic content of states; that is, no knowledge is lost or acquired as a result of this action. This is formalised in the next two results, where we write $F:$ Set $\rightarrow$ Set for the functor defined by $F(S)=\mathcal{P}_{\kappa}(S)^{A g} \times \mathcal{P}(A t)$.

Proposition 3. In any appearance-update coalgebra $(S,\langle a p$, up, val $\rangle)$ where the set Ac of actions includes the $\tau$ action, up $(s)(\tau) \sim_{F} s$ for any state $s \in S$, where $\sim_{F} \subseteq S \times S$ denotes the $F$-bisimilarity relation on the $F$-coalgebra $(S,\langle$ ap, val $\rangle)$.

Proof. The statement follows by coinduction, namely by showing that the relation $R \subseteq S \times S$ given by $\{(s, u p(s)(\tau)) \mid s \in S\}$ is an $F$-bisimulation. The preservation of facts ensures that $R$ only relates states with the same valuations, whereas the rationality restriction guarantees closure of $R$ under appearances.

Since $F$-bisimilar states satisfy the same content formulas, a stronger preservation of knowledge result can now be formulated for the $\tau$ action.

Corollary 1. Let $(S,\langle a p$, up, val $\rangle)$ be an appearance-update coalgebra. Then for all contents $\mu$ and all states $s \in S$, we have

$$
s \models \mu \quad \Longleftrightarrow \quad u p(s)(\tau) \models \mu
$$

Public Announcements. The public announcement of a content $\mu$ is denoted $\mu$ !, and has $A c_{\mu !, A}=\{\mu !\}$ for all $A \in A g$. We define truthful common knowledge of a content $\mu$ among a group $\beta$ of agents as follows

$$
C K_{\beta} \mu:=\bigwedge_{\left\langle A_{0}, A_{1}, \ldots, A_{n}\right\rangle \in \beta^{*}} \square_{A_{0}} \square_{A_{1}} \ldots \square_{A_{n}} \mu
$$

where $\beta^{*}=\cup_{i \in \mathbb{N}} \beta^{i}$ is the set of all finite sequences of agents in $\beta$, including the empty sequence. Excluding the empty sequence provides us with the notion of not necessarily truthful common knowledge, denoted $\square_{\beta}^{*} \mu$.

We now show that the public announcement of a positive content results in truthful common knowledge of that content.

\footnotetext{
${ }^{6}$ To be in line with the existing literature, we consider contents rather than preconditions of actions. The difference between the two is best seen in an example: the content of a public announcement is simply the announced proposition $\mu$, whereas its precondition is the conjunction of $\mu$ with the knowledge of the announcer about $\mu$.
} 
Proposition 4. For a state $s$ of an appearance-update coalgebra in which the public announcement $\mu$ ! with positive $\mu$ is possible, we have up $(s)(\mu !) \models C K_{A g} \mu$.

Proof. We must show that for any state $s$ and any state $s^{\prime}$ connected to the applicable update $u p(s)(\mu !) \neq *$ via any sequence of appearance maps we have $s^{\prime} \models \mu$. Thus, we have a sequence of states $u p(s)(\mu !)=s_{0}, s_{1}, \ldots, s_{m}=s^{\prime}$ such that for $0 \leq j<m$ and some agent $A_{j} \in A g$ we have $s_{j+1} \in a p\left(s_{j}\right)\left(A_{j}\right)$. For $m=0, s_{0} \models \mu$ follows from the applicability of update $u p(s)(\mu !) \neq *$, the content restriction, and the preservation result. For $m>0$, we have that $s_{m}$ is in the following set of nested appearances

$$
a p\left(\ldots\left(\operatorname{ap}\left(\operatorname{ap}(u p(s)(\mu !))\left(A_{0}\right)\right)\left(A_{1}\right)\right) \ldots\right)\left(A_{m-1}\right)
$$

which, by applying the rationality restriction $m$ times, is equal to

$$
\begin{array}{r}
\left\{u p\left(t_{m}\right)(\mu !) \mid t_{m} \in \operatorname{ap}\left(t_{m-1}\right)\left(A_{m-1}\right), \ldots, t_{2} \in a p\left(t_{1}\right)\left(A_{1}\right), t_{1} \in a p(s)\left(A_{0}\right),\right. \\
\text { and } \left.u p\left(t_{1}\right)(\mu !) \neq *, u p\left(t_{2}\right)(\mu !) \neq *, \ldots, u p\left(t_{m}\right)(\mu !) \neq *\right\}
\end{array}
$$

By the content restriction $u p\left(t_{m}\right)(\mu !) \neq *$ is equivalent to $t_{m} \models \mu$, and from this by the preservation result it follows that $u p\left(t_{m}\right)(\mu !) \models \mu$.

The closest special case to this proposition is that of $[3$, where the authors show that common knowledge of a fact implies preservation of any content under the public announcement of that fact.

Private Announcements. A private announcement $\mu !_{\beta}$ is the action of announcing the content $\mu$ to a subgroup of agents $\beta \subseteq A g$ with $A c_{\mu !_{\beta}, B}=\left\{\mu !_{\beta}\right\}$ for $B \in \beta$ and $A c_{\mu !}, A=\{\tau\}$ for $A \notin \beta$.

As expected, one can prove that the private announcement of a positive content to a subgroup of agents results in truthful common knowledge of that content among the subgroup, and has no visible effect outside the subgroup.

Proposition 5. For $\beta \subseteq A g$ and a state $s$ of an appearance-update coalgebra in which the private announcement $\mu !_{\beta}$ with positive $\mu$ is possible, we have up $(s)\left(\mu !_{\beta}\right) \models C K_{\beta} \mu$ and ap $\left(u p(s)\left(\mu !_{\beta}\right)\right)(A) \sim^{\mathcal{P}}$ ap $(s)(A)$ for $A \notin \beta$ ㄱ.

Lying. We write $\mu \dagger_{A}$ for the action with content $\neg \mu$ in which an agent $A$ lies that $\mu$ to the rest of the agents. We have $A c_{\mu \dagger_{A}, A}=\left\{\mu \dagger_{A}\right\}$ and $A c_{\mu \dagger_{A}, B}=\{\mu !\}$ for any $B \neq A$.

Proposition 6. For any agent $A \in A g, \beta=A g \backslash\{A\}$, and any state $s$ of an appearance-update coalgebra in which the lying action $\mu_{A_{A}}$ with a positive $\mu$ is possible, we have up $(s)\left(\mu \dagger_{A}\right) \models \square_{\beta}^{*} \mu$ and up $(s)\left(\mu \dagger_{A}\right) \models \square_{A} \square_{\beta}^{*} \mu$.

Proof. In order to show that $u p(s)\left(\mu_{A}\right) \models \square_{\beta}^{*} \mu$, we must show that for any state $s$ and any state $s^{\prime}$ connected to the applicable update $u p(s)\left(\mu_{\dagger_{A}}\right) \neq *$

\footnotetext{
${ }^{7}$ Here $\sim^{\mathcal{P}}$ denotes the lifting of the bisimilarity relation on $S$ to $\mathcal{P}(S)$, see e.g. [12.
} 
via any sequence of length more than 1 of appearance maps of agents in $\beta$, we have $s^{\prime} \models \mu$. Consider a sequence of states $u p(s)\left(\mu \dagger_{A}\right)=s_{0}, s_{1}, \ldots, s_{m}=s^{\prime}$ with $1 \leq m$, such that for $0 \leq j<m$ and some agent $B_{j} \in A g \backslash\{A\}$ we have $s_{j+1} \in \overline{a p}\left(s_{j}\right)\left(B_{j}\right)$. It follows that $s_{m}$ is in the following set of nested appearances

$$
\operatorname{ap}\left(\ldots\left(\operatorname{ap}\left(\operatorname{ap}\left(u p(s)\left(\mu \dagger_{A}\right)\right)\left(B_{0}\right)\right)\left(B_{1}\right)\right) \ldots\right)\left(B_{m-1}\right)
$$

which, by applying the rationality restriction $m$ times (once for the lying action $\mu \dagger_{A}$ and $B_{0}$ and $m-1$ times for the public announcement $\mu$ ! and $B_{1}$ to $\left.B_{m-1}\right)$, is equal to

$$
\begin{array}{r}
\left\{u p\left(t_{m}\right)(\mu !) \mid t_{m} \in a p\left(t_{m-1}\right)\left(B_{m-1}\right), \ldots, t_{2} \in \operatorname{ap}\left(t_{1}\right)\left(B_{1}\right), t_{1} \in a p(s)\left(B_{0}\right),\right. \\
\text { and } \left.u p\left(t_{1}\right)(\mu !) \neq *, u p\left(t_{2}\right)(\mu !) \neq *, \ldots, u p\left(t_{m}\right)(\mu !) \neq *\right\}
\end{array}
$$

By the content restriction $u p\left(t_{m}\right)(\mu !) \neq *$ is equivalent to $t_{m} \models \mu$, and from this by the preservation result it follows that $u p\left(t_{m}\right)(\mu !) \models \mu$.

Now to show that $u p(s)\left(\mu \dagger_{A}\right) \models \square_{A} \square_{\beta}^{*} \mu$, we must show that $t \models \square_{\beta}^{*} \mu$ for all $t \in \operatorname{ap}\left(u p(s)\left(\mu \dagger_{A}\right)\right)(A)$. By the rationality restriction we have

$$
\operatorname{ap}\left(u p(s)\left(\mu \dagger_{A}\right)\right)(A)=\left\{u p(w)\left(\mu \dagger_{A}\right) \mid w \in \operatorname{ap}(s)(A), u p(w)\left(\mu \dagger_{A}\right) \neq *\right\}
$$

Since $u p(w)\left(\mu \dagger_{A}\right) \neq *$ and $\mu$ is positive, it follows from $u p(s)\left(\mu \dagger_{A}\right) \models \square_{\beta}^{*} \mu$ that $u p(w)\left(\mu \dagger_{A}\right) \models \square_{\beta}^{*} \mu$.

Security Actions. A security action $\mu \star \mu_{\{A\}, \beta, \gamma}^{\prime}$ is a private announcement in an unsafe communication channel, where the intruders in $\gamma$ change the original content $\mu$, sent by $A$ to the agents in $\beta$, to a fake one $\mu^{\prime}$. In this case we have $A c_{\mu \star \mu_{\{A\}, \beta, \gamma}^{\prime}, A}=\left\{\mu !_{\beta \cup\{A\}}\right\}, A c_{\mu \star \mu_{\{A\}, \beta, \gamma}^{\prime}, B}=\left\{\mu^{\prime} !_{\beta \cup\{A\}}\right\}$ for agents $B \in \beta$, $A c_{\mu \star \mu_{\{A\}, \beta, \gamma}^{\prime}, C}=\left\{\mu \star \mu_{\{A\}, \beta, \gamma}^{\prime}\right\}$ for the intruders $C \in \gamma$, while $A c_{\mu \star \mu_{\{A\}, \beta, \gamma}^{\prime}, D}=$ $\{\tau\}$ for any other agent $D \in A g \backslash(\{A\} \cup \beta \cup \gamma)$.

Proposition 7. For any agents $B \in \beta, C \in \gamma$, and any state $s$ of an appearanceupdate coalgebra in which the security action $\mu \star \mu_{\{A\}, \beta, \gamma}^{\prime}$ with positive $\mu$ and $\mu^{\prime}$ is possible, we have up $(s)\left(\mu \star \mu_{\{A\}, \beta, \gamma}^{\prime}\right) \models \square_{A} C K_{\beta} \mu, u p(s)\left(\mu \star \mu_{\{A\}, \beta, \gamma}^{\prime}\right) \models \square_{\beta}^{*} \square_{A} \mu^{\prime}$ and $u p(s)\left(\mu \star \mu_{\{A\}, \beta, \gamma}^{\prime}\right) \models C K_{\gamma}\left(\square_{A} C K_{\beta} \mu \wedge \square_{\beta}^{*} \square_{A} \mu^{\prime}\right)$.

\section{Comparison with Baltag's Coalgebraic Model}

We now compare our coalgebraic semantics with that of 11. In loc. cit., both epistemic states and epistemic actions are defined via final coalgebras. Two different functors of a similar shape are used to achieve this. However, none of these functors accounts for epistemic updates, which are instead modelled using a partial product between coalgebras of states and coalgebras of actions.

The functor used in [1] to model epistemic states is

$$
F: S e t \rightarrow S e t, \quad F(S)=\mathcal{P}_{\kappa}(S)^{A g} \times \mathcal{P}(A t)
$$


Appearances of states to agents are encoded as elements of $\mathcal{P}_{\kappa}(S)^{A g}$, while their valuations are encoded using sets of atomic propositions. Epistemic states are then defined as elements of the final $F$-coalgebra $\Psi$. Similarly, epistemic actions are defined as elements of the final coalgebra of the functor

$$
G: \text { Set } \rightarrow \text { Set }, \quad G(\Sigma)=\mathcal{P}_{\kappa}(\Sigma)^{A g} \times \mathcal{P}(\Psi)
$$

with $\mathcal{P}_{\kappa}(\Sigma)^{A g}$ encoding the appearances of actions to agents, and $\mathcal{P}(\Psi)$ encoding the contents of actions (as sets of epistemic states where the actions are applicable). Finally, epistemic updates are modelled using a functor

$$
-\otimes-: \operatorname{Coalg}(F) \times \operatorname{Coalg}(G) \rightarrow \operatorname{Coalg}(F)
$$

which takes a pair consisting of an $F$-coalgebra $\left(S,\left\langle a p_{S}, v a l_{S}\right\rangle\right)$ and a $G$-coalgebra $\left(\Sigma,\left\langle a p_{\Sigma}\right.\right.$, cont $\left.\left._{\Sigma}\right\rangle\right)$ to another $F$-coalgebra whose elements correspond to updates of states in $S$ with actions in $\Sigma$. Writing $!_{S}: S \rightarrow \Psi$ for the unique $F$-coalgebra morphism arising from the finality of $\Psi$, the coalgebra for the updated states has carrier

$$
S \otimes \Sigma=\left\{(s, \sigma) \in S \times \Sigma \mid !_{S}(s) \in \operatorname{cont}_{\Sigma}(\sigma)\right\}
$$

That is, updated states are pairs consisting of a state $s \in S$ and an action $\sigma \in \Sigma$, with the additional property that the content of the action $\sigma$ makes it applicable to the state 8 . The coalgebra map $\left\langle a p_{S \otimes \Sigma}, v a l_{S \otimes \Sigma}\right\rangle: S \otimes \Sigma \rightarrow F(S \otimes \Sigma)$ is given by

$$
\begin{aligned}
a p_{S \otimes \Sigma}(s, \sigma)(A) & =\left\{\left(s^{\prime}, \sigma^{\prime}\right) \in S \otimes \Sigma \mid s^{\prime} \in a p_{S}(s)(A), \sigma^{\prime} \in a p_{\Sigma}(\sigma)(A)\right\} \\
v a l_{S \otimes \Sigma}(s, \sigma) & =\operatorname{val}_{S}(s)
\end{aligned}
$$

That is, the appearances of updated states to agents are computed using both the appearances of the original states and the appearances of the actions producing the updates.

In contrast to the above, our approach uses only one functor, which incorporates both the epistemic and the dynamic aspect of states. This internal modelling of updates is made possible by the fact that we apriorily fix a universe $A c$ of actions, together with its epistemic structure. The set $A c$ should be taken to contain those epistemic actions (elements of the final $G$-coalgebra) which are of interest to the modelling of a particular multi-agent scenario. In this setting, our choice to specify for each action $a \in A c$ and agent $A \in A g$, a set $A c_{a, A}$ of actions that are perceived by $A$, together with for each action $a$ a content $\mu_{a}$, gives rise to a coalgebra $\left(A c,\left\langle a p_{A c}, \mu_{A c}\right\rangle\right)$ of the following functor

$$
H: \text { Set } \rightarrow \text { Set }, \quad H(\Sigma)=\mathcal{P}_{\kappa}(\Sigma)^{A g} \times C
$$

where the set $C$ consists of equivalence classes of content formulas. Here, two content formulas are said to be (semantically) equivalent if they are satisfied by

\footnotetext{
${ }^{8}$ Here it is assumed that the applicability of an action is invariant under bisimulation, and therefore an action is applicable to a state precisely when it is applicable to its image under the unique coalgebra morphism into the final $F$-coalgebra.
} 
the same states of any $F$-coalgebra. The map $a p_{A c}$ of the previously mentioned $H$-coalgebra is given by $a p_{A c}(a)(A)=A c_{a, A}$ for $a \in A c$ and $A \in A g$, whereas the map $\mu_{A c}$ takes actions $a \in A c$ to the equivalence class of their content $\left[\mu_{a}\right]$. In this way, we do not distinguish between actions that have both the same epistemic structure and semantically equivalent contents.

In order to make precise the relationship between appearance-update $T$ coalgebras and the models of [1, we make the dependency of $T$ on the set $A c$ of actions explicit, and write $T_{A c}:$ Set $\rightarrow$ Set for the functor given by

$$
T_{A c} X=\left(\mathcal{P}_{\kappa} X\right)^{A g} \times(1+X)^{A c} \times \mathcal{P}(A t)
$$

Next, we let $A p p U p C o a l g$ denote the category whose objects are pairs $(A c, S)$, with $A c=\left(A c,\left\langle a p_{A c}, \mu_{A c}\right\rangle\right)$ an $H$-coalgebra and $S=\left(S,\left\langle a p_{S}, u p_{S}, v a l_{S}\right\rangle\right)$ an appearance-update $T_{A c}$-coalgebra. The $H$-coalgebra $A c$ encodes the structure on the set $A c$ of actions required to formulate the content and rationality restrictions

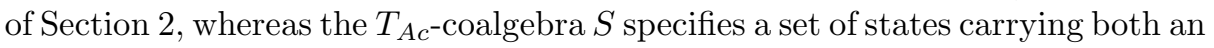
epistemic structure and a dynamic structure w.r.t. the actions in $A c$. To define the arrows of the category $A p p U p C o a l g$, we first note that any $H$-coalgebra morphism $f: A c \rightarrow A c^{\prime}$ induces a functor

$$
U_{f}: \operatorname{Coalg}\left(T_{A c^{\prime}}\right) \rightarrow \operatorname{Coalg}\left(T_{A c}\right)
$$

which takes a $T_{A c^{\prime}}$-coalgebra $\left(S,\left\langle a p_{S}, u p_{S}, v a l_{S}\right\rangle\right)$ to the $T_{A c^{-}}$coalgebra with the same carrier set and appearance and valuation maps, but with an update map w.r.t. the set $A c$ instead. This update is derived from the curried version $e v\left(u p_{S}\right): S \times A c^{\prime} \rightarrow(1+S)$ of the update map $u p_{S}$ of the $T_{A c^{\prime}}$-coalgebra, as shown below

$$
S \times A c \stackrel{i d_{S} \times f}{\longrightarrow} S \times A c^{\prime} \stackrel{e v\left(u p_{S}\right)}{\longrightarrow} 1+S
$$

The curried version of this composition is the update map of the $T_{A c}$-coalgebra

$$
e v\left(e v\left(u p_{S}\right) \circ\left(i d_{S} \times f\right)\right): S \rightarrow(1+S)^{A c}
$$

So we have $U_{f}\left(S,\left\langle a p_{S}, u p_{S}, v a l_{S}\right\rangle\right)=\left(S,\left\langle a p_{S}, e v\left(e v\left(u p_{S}\right) \circ\left(i d_{S} \times f\right)\right), v a l_{S}\right\rangle\right)$. Now the arrows from $(A c, S)$ to $\left(A c^{\prime}, S^{\prime}\right)$ in the category AppUpCoalg are pairs of maps $(f, g)$ with $f: A c \rightarrow A c^{\prime}$ an $H$-coalgebra morphism and $g: S \rightarrow U_{f} S^{\prime}$ a $T_{A c^{-}}$-coalgebra morphism. The former encodes the actions in $A c$ as actions in $A c^{\prime}$, whereas the latter translates the states of the $T_{A c^{-}}$coalgebra $S$ to states of the $T_{A c^{\prime}}$-coalgebra $S^{\prime}$.

The last piece of notation we require before relating our models to those of [1] concerns characteristic formulas for states of $F$-coalgebras. These are infinitary formulas of the form used in Section2 to specify the contents of epistemic actions, and have the additional property that they characterise individual states of $F$ coalgebras up to bisimulation. Their existence is guaranteed by the $\kappa$-accessibility of $F$. In particular, for any state $\psi$ of the final $F$-coalgebra $\Psi$, there exists a characteristic formula $\phi_{\psi}$ with the property that, given any state $s$ of an $F$ coalgebra $S$, we have $s \models \phi_{\psi}$ if and only if $!_{S}(s)=\psi$. 
We are now ready to describe the relationship between the models of [1] and our appearance-update coalgebras. This is given by an adjunction

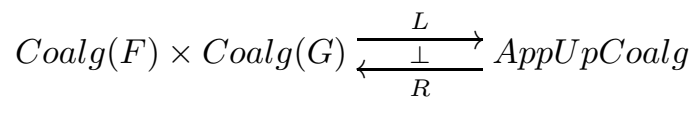

Definition 2 (Left adjoint). We let $L: \operatorname{Coalg}(F) \times \operatorname{Coalg}(G) \rightarrow$ AppUpCoalg be defined by $L(S, \Sigma)=\left(\left(\Sigma,\left\langle a p_{\Sigma}, \mu_{\Sigma}\right\rangle\right),\left(S^{\prime},\left\langle a p_{S^{\prime}}, u p_{S^{\prime}}, v a l_{S^{\prime}}\right\rangle\right)\right)$, where

$-\mu_{\Sigma}(\sigma)=\bigvee_{\psi \in \operatorname{cont}_{\Sigma}(\sigma)} \phi_{\psi}$, where for $\psi \in \Psi, \phi_{\psi}$ is the characteristic formula of $\psi$.

- $S^{\prime}=\left(S^{\prime},\left\langle a p_{S^{\prime}}, u p_{S^{\prime}}, v a l_{S^{\prime}}\right\rangle\right)$ is a $T_{\Sigma^{-}}$-coalgebra obtained by

1. first letting $S^{\prime}=\left(S^{\prime},\left\langle a p_{S^{\prime}}, v^{\prime} a l_{S^{\prime}}\right\rangle\right)=\cup_{i \in \omega}\left(S_{i},\left\langle a p_{S_{i}}, v a l_{S_{i}}\right\rangle\right)$ where

$$
S_{0}=S, \quad S_{i+1}=S_{i} \otimes \Sigma \quad \text { for } i \in \omega
$$

(Note that, by definition, each of the sets $S_{i}$ comes equipped with an F-coalgebra structure, and $S^{\prime}$ inherits this structure.)

2. subsequently endowing the set $S^{\prime}$ with an update map up p $_{S^{\prime}}: S^{\prime} \rightarrow(1+$ $\left.S^{\prime}\right)^{\Sigma}$, by letting

$$
u_{S^{\prime}}\left(s_{i}\right)(\sigma)=\left\{\begin{array}{ll}
\left(s_{i}, \sigma\right) & \text { if }\left(s_{i}, \sigma\right) \in S_{i+1} \\
* & \text { otherwise }
\end{array}, \quad \text { for } i \in \omega\right.
$$

In informal terms, the functor $L$ constructs an $H$-coalgebra $\Sigma$ and a $T_{\Sigma^{-}}$ coalgebra $S^{\prime}$ from a pair consisting of an $F$-coalgebra $S$ and a $G$-coalgebra $\Sigma$. The $H$-structure of $\Sigma$ is determined by the $G$-structure of $\Sigma$ in a trivial way: appearances of actions to agents are already defined by the $H$-structure, whereas the content map $\mu_{\Sigma}: \Sigma \rightarrow C$ acts on an action $\sigma \in \Sigma$ by logically joining all the characteristic formulas of states in the content of $\sigma$. The $T_{\Sigma}$-coalgebra $S^{\prime}$ is obtained by performing consecutive update products with the actions in $\Sigma$, first on $S$, and then on the result of the preceding update product:

$$
S \longmapsto S \otimes \Sigma \longmapsto(S \otimes \Sigma) \otimes \Sigma \longmapsto \ldots
$$

and subsequently taking the union of the resulting $F$-coalgebras and endowing it with an update map.

Proposition 8. The $T_{\Sigma}$-coalgebra $S^{\prime}$ is an appearance-update coalgebra.

Proof. We have to show that $S^{\prime}$ satisfies all the restrictions in $\mathcal{R}$. The preservation of facts follows directly from the definitions of $S_{i}$ and $S^{\prime}$ : for $i \in \omega$, whenever $u p_{S^{\prime}}\left(s_{i}\right)(\sigma) \in S^{\prime}$, that is, whenever $\left(s_{i}, \sigma\right) \in S_{i+1}$, we have

$$
\operatorname{val}_{S^{\prime}}\left(u p_{S^{\prime}}\left(s_{i}\right)(\sigma)\right)=\operatorname{val}_{S_{i+1}}\left(s_{i}, \sigma\right)=\operatorname{val}_{S_{i}}\left(s_{i}\right)=\operatorname{val}_{S^{\prime}}\left(s_{i}\right)
$$

For the rationality restriction, assuming $u p_{S^{\prime}}\left(s_{i}\right)(\sigma) \in S^{\prime}$, that is, $\left(s_{i}, \sigma\right) \in S_{i+1}$, we have 


$$
\begin{aligned}
a p_{S^{\prime}}\left(u p_{S^{\prime}}\left(s_{i}\right)(\sigma)\right)(A) & =a p_{S_{i+1}}\left(s_{i}, \sigma\right)(A) \\
& =\left\{\left(s^{\prime}, \sigma^{\prime}\right) \in S_{i} \otimes \Sigma \mid s^{\prime} \in a p_{S_{i}}\left(s_{i}\right)(A), \sigma^{\prime} \in a p_{\Sigma}(\sigma)(A)\right\}
\end{aligned}
$$

and

$$
\begin{array}{r}
\left\{u p_{S^{\prime}}(t)\left(\sigma^{\prime}\right) \mid t \in a p_{S^{\prime}}\left(s_{i}\right)(A), \sigma^{\prime} \in a p_{\Sigma}(\sigma)(A), u p_{S^{\prime}}(t)\left(\sigma^{\prime}\right) \neq *\right\}= \\
\left\{\left(t, \sigma^{\prime}\right) \mid t \in a p_{S_{i}}\left(s_{i}\right)(A), \sigma^{\prime} \in a p_{\Sigma}(\sigma)(A),\left(t, \sigma^{\prime}\right) \in S_{i+1}\right\}= \\
\left\{\left(t, \sigma^{\prime}\right) \in S_{i} \otimes \Sigma \mid t \in a p_{S_{i}}\left(s_{i}\right)(A), \sigma^{\prime} \in a p_{\Sigma}(\sigma)(A)\right\}
\end{array}
$$

for each $i \in \omega$, and therefore

$$
\begin{aligned}
& a p_{S^{\prime}}\left(u p_{S^{\prime}}\left(s^{\prime}\right)(\sigma)\right)(A)= \\
& \quad\left\{u p_{S^{\prime}}(t)\left(\sigma^{\prime}\right) \mid t \in a p_{S^{\prime}}\left(s^{\prime}\right)(A), \sigma^{\prime} \in a p_{\Sigma}(\sigma)(A), u p_{S^{\prime}}(t)\left(\sigma^{\prime}\right) \neq *\right\}
\end{aligned}
$$

Finally, for the content restriction, we have, for each $i \in \omega$

$$
\begin{array}{ccc}
u p_{S^{\prime}}\left(s_{i}\right)(\sigma) \in S^{\prime} & \text { iff } \quad\left(s_{i}, \sigma\right) \in S_{i+1}=S_{i} \otimes \Sigma & \text { iff } \\
!_{S_{i}}\left(s_{i}\right) \in \operatorname{cont}_{\Sigma}(\sigma) & \text { iff } \quad !_{S_{i}}\left(s_{i}\right) \models \mu_{\Sigma}(\sigma) & \text { iff } \\
!_{S^{\prime}}\left(s_{i}\right) \models \mu_{\Sigma}(\sigma) & \text { iff } & s_{i} \models \mu_{\Sigma}(\sigma)
\end{array}
$$

and hence $u p_{S^{\prime}}\left(s_{i}\right)(\sigma) \in S^{\prime}$ iff $s_{i} \models \mu_{\Sigma}(\sigma)$.

Definition 3 (Right adjoint). We define $R: \operatorname{App} U p \operatorname{Coalg} \rightarrow \operatorname{Coalg}(F) \times$ $\operatorname{Coalg}(G)$ by $R(A c, S)=\left(\left(S,\left\langle a p_{S}, v_{a l}\right\rangle\right),\left(A c,\left\langle a p_{A c}\right.\right.\right.$, cont $\left.\left._{A c}\right\rangle\right)$, where the map cont $_{A c}: A c \rightarrow \mathcal{P}(\Psi)$ takes an action $a \in A c$ to the set of states in the final $F$-coalgebra which satisfy the formula $\mu_{A c}(a)$.

Informally speaking, the functor $R$ takes a pair consisting of an $H$-coalgebra $A c$ and an appearance-update $T_{A c^{-}}$-coalgebra $S$, and produces an $F$-coalgebra and a $G$-coalgebra. The $F$-coalgebra is obtained from $S$ by forgetting its update map and keeping everything else intact. The $G$-coalgebra has the same carrier set and epistemic structure as $A c$, and a content map obtained essentially by replacing content formulas with their denotations in the final $F$-coalgebra.

Theorem 1. L is left adjoint to $R$.

Proof. We begin by examining the unit and counit of this adjunction. Since the categories $\operatorname{Coalg}(H)$ and $\operatorname{Coalg}(G)$ are naturally isomorphic, it is the move from $T_{A c}$-coalgebras to $F$-coalgebras and back that makes the adjunction non-trivial.

For the unit of the adjunction, the inclusions $\eta_{S, \Sigma}: S \rightarrow S \cup(S \otimes \Sigma) \cup((S \otimes$ $\Sigma) \otimes \Sigma) \cup \ldots$ together with the natural isomorphism between $\operatorname{Coalg}(H)$ and $\operatorname{Coalg}(G)$ give rise to a natural transformation $\eta: I_{\operatorname{Coalg}(F) \times \operatorname{Coalg}(G)} \Rightarrow R \circ L$.

For the counit, the maps $\epsilon_{A c, S}: S \cup(S \otimes A c) \cup((S \otimes A c) \otimes A c) \cup \ldots \rightarrow S$ defined inductively by

$$
\epsilon_{A c, S}(s)=s, \quad \epsilon_{A c, S}\left(s_{i}, a\right)=u p_{S}\left(\epsilon_{A c, S}(s)\right)(a) \quad \text { for } i \in \omega \text { and } s_{i} \in S_{i}
$$

together with the natural isomorphism between $\operatorname{Coalg}(H)$ and $\operatorname{Coalg}(G)$, yield a natural transformation $\epsilon: L \circ R \Rightarrow I d_{A p p U p C o a l g}$. 
We show that $\eta$ and $\epsilon$ indeed constitute the unit and counit of an adjunction $L \dashv R$. To this end, we fix $(S, \Sigma) \in \operatorname{Coalg}(F) \times \operatorname{Coalg}(G)$ and $\left(A c, S^{\prime}\right) \in$ AppUpCoalg. For $(f, g):(S, \Sigma) \rightarrow R\left(A c, S^{\prime}\right)$, the map $f^{\#}: S \cup(S \otimes \Sigma) \cup((S \otimes$ $\Sigma) \otimes \Sigma) \cup \ldots \rightarrow S^{\prime}$ defined inductively by

$$
f^{\#}(s)=f(s), \quad f^{\#}\left(s_{i}, \sigma\right)=u p_{S^{\prime}}\left(f^{\#}\left(s_{i}\right)\right)(g(\sigma)) \quad \text { for } i \in \omega
$$

is a $T_{A c^{-}}$-coalgebra morphism that satisfies $R\left(g, f^{\#}\right) \circ \eta_{(S, \Sigma)}=(f, g)$. Furthermore, any $T_{A c}$-coalgebra morphism with the above property is defined in this way. For $(h, k): L(S, \Sigma) \rightarrow\left(A c, S^{\prime}\right)$, the map $k^{b}: S \rightarrow S^{\prime}$ given by $k \uparrow_{S}$ defines an $F$-coalgebra morphism that satisfies $\epsilon_{\left(A c, S^{\prime}\right)} \circ L\left(k^{b}, h\right)=(h, k)$. Furthermore, this last requirement uniquely determines the definition of $k^{b}$.

\section{Coalgebraic Dynamic Epistemic Logic}

Coalgebras give rise to modal logics in different ways, for example the coalgebraic logic of Moss [13, the temporal logic of Jacobs [11, and the modular logic of Cîrstea and Pattinson [5]6. In previous work [15], we showed how one obtains an algebraic logic from our functor by predicate lifting, and investigated the connection between this logic and the algebraic dynamic epistemic logic of 2114. Cîrstea and Pattinson have shown how complete and expressive coalgebraic logics can be derived in a modular fashion for an inductively-defined class of endofunctors on Set. By applying this method to our setting, we obtain a logic with a multi-sorted syntax, which is expressive - that is, two states are bisimilar if and only if they satisfy the same formulas -, and admits a sound and complete proof system. Because of the particular shape of the functor $\mathrm{T}$ and of the axioms in the associated proof system, the multi-sorted syntax of this logic can be simplified to the following single-sorted syntax, with no loss in expressiveness

$$
\phi::=t t|p| \neg \phi|\phi \wedge \phi| \square_{A} \phi \mid[a] \phi
$$

The standard knowledge and dynamic modalities, that is, $\square_{A}$ (to be read as ' $A$ knows $\phi^{\prime}$ ) and $[a]$ (to be read as 'after $a, \phi^{\prime}$ ), are recovered by letting $\square_{A} \phi::=$ $\left[\pi_{1}\right][A] \square \phi$ and $[a] \phi::=\left[\pi_{2}\right][a]\left[\kappa_{2}\right] \phi 9$. In particular, the statement 'action $a$ does not go through' is captured by the formula $[a]$ ff. Using the simplified syntax, the original proof system is equivalent to the following set of axioms and rules

$$
\vdash \bigcirc t t \quad \vdash \bigcirc \phi \wedge \bigcirc \psi \rightarrow \bigcirc(\phi \wedge \psi) \quad \frac{\vdash \phi \rightarrow \psi}{\vdash \bigcirc \phi \rightarrow \bigcirc \psi}
$$

for $\bigcirc \in\left\{\square_{A},[a]\right\}$, and

$$
\vdash[a](\phi \vee \psi) \rightarrow[a] \phi \vee[a] \psi
$$

on top of propositional logi 10 . As a consequence of the results in [6], this proof system is sound and complete w.r.t. $T$-coalgebras. However, in order to formulate

\footnotetext{
${ }^{9}$ See [6] for details of the multi-sorted syntax.

${ }^{10}$ As in [6], we include all instances of propositional tautologies and the modus ponens rule into our set of axioms and rules.
} 
a soundness and completeness result w.r.t. appearance-update coalgebras, the restrictions defining appearance-update coalgebras must also be axiomatised. To this end, we add the following axioms to the previous proof system:

$$
\begin{aligned}
\vdash[a] p \leftrightarrow(\neg[a] \mathrm{ff} \rightarrow p) & \vdash[a] \square_{A} \phi \leftrightarrow\left(\neg[a] \mathrm{ff} \rightarrow \bigwedge_{a^{\prime} \in A c_{a, A}} \square_{A}\left[a^{\prime}\right] \phi\right) \\
& \phi_{a} \leftrightarrow \neg[a] \mathrm{ff}
\end{aligned}
$$

where for an action $a \in A c$, its content is denoted by $\phi_{a}$. There is one such axiom for each epistemic action $a$ and each (type of) agent $A$.

Example of Derivation. Consider a simple Man in the Middle Attack: agent $A$ sends a message with factual content $p$ to agent $B$, but on the way the intruder $C$ changes $p$ to another fact $p^{\prime}$ and thus $B$ receives $p^{\prime}$ instead. If we assume that $A$ does not suspect the interception, after sending $p$ he believes that $B$ believes in $p$. Similarly, upon receipt, $B$ believes that $A$ believes in $p^{\prime}$. In security terms and since $A$ and $B$ do not suspect the interception, they will wrongly authenticate with each other. We use the encoding of the security action in Section 2 to prove that $\vdash\left[p \star p_{A, B, C}^{\prime}\right] \square_{A} \square_{B} p$. The proof steps are sketched below:

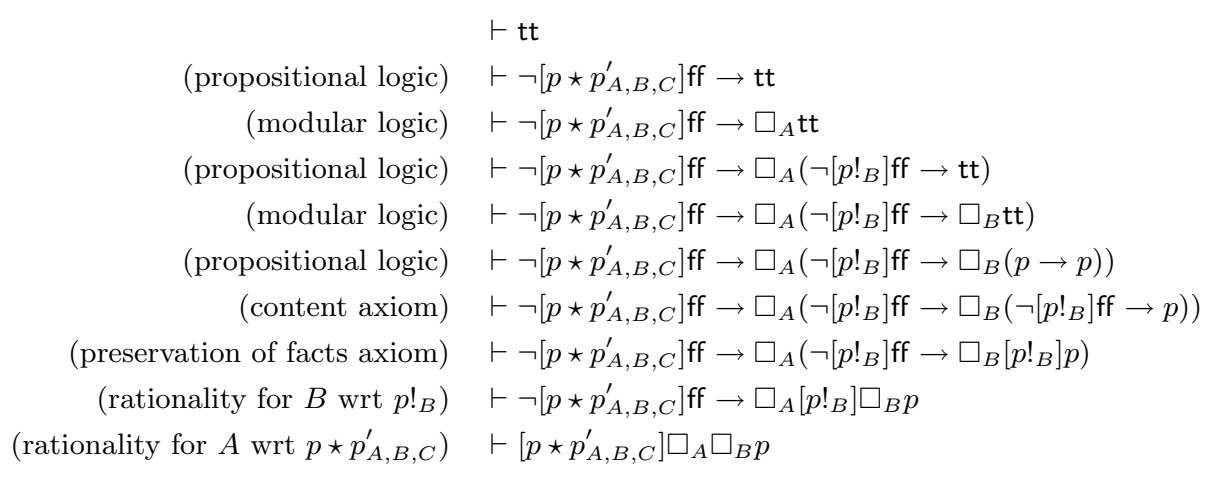

With the additional axioms, we obtain the following result:

Theorem 2 (Soundness and Completeness). A formula holds in all appearance-update coalgebras if and only if it is derivable in the appearanceupdate logic.

Proof. The proof of both soundness and completeness is detailed in [8]. Here we only sketch the completeness proof. This follows the same line as the completeness result for dynamic epistemic logic [3], and is based on a translation between our appearance-update logic (with appearance-update coalgebras as models) and ordinary epistemic logic (with $F$-coalgebras as models). As in [3], this translation has the property that a formula $\phi$ is semantically equivalent to its translation $\phi^{t}$. Moreover, the axioms and rules of appearance-update logic ensure that $\vdash \phi \leftrightarrow \phi^{t}$. These properties, together with our result in [8] that the 
final $F$-coalgebra can be extended to an appearance-update coalgebra, allow us to make use of the completeness result of [6] for $F$-coalgebras in order to prove completeness of appearance-update logic w.r.t. appearance-update coalgebras.

Acknowledgement. We would like to thank the anonymous referees for valuable suggestions on improving the paper.

\section{References}

1. Baltag, A.: A coalgebraic semantics for epistemic programs. In: Proceedings of Coalgebraic Methods in Computer Science. Electronic Notes in Theoretical Computer Science, vol. 82 (2003)

2. Baltag, A., Coecke, B., Sadrzadeh, M.: Epistemic actions as resources. Journal of Logic and Computation, forthcoming

3. Baltag, A., Moss, L.S.: Logics for epistemic programs. Synthese 139 (2004)

4. van Benthem, J., Pacuit, E.: The tree of knowledge in action: towards a common perspective. In: Proceedings of Advances in Modal Logic (2006)

5. Cîrstea, C.: A compositional approach to defining logics for coalgebras. Theoretical Computer Science 327(1), 45-69 (2004)

6. Cîrstea, C., Pattinson, D.: Modular construction of modal logics. In: Gardner, P., Yoshida, N. (eds.) CONCUR 2004. LNCS, vol. 3170, pp. 258-275. Springer, Heidelberg (2004)

7. Cîrstea, C.: On expressivity and compositionality in logics for coalgebras. In: Proceedings of Coalgebraic Methods in Computer Science, Electronic Notes in Theoretical Computer Science 82 (2003)

8. Cîrstea, C., Sadrzadeh, M.: Coalgebraic epistemic update without change of model http://ecs.soton.ac.uk/ ms6/TechRep.pdf

9. Fagin, R., Halpern, J.Y., Moses, Y., Vardi, M.Y.: Reasoning about Knowledge. MIT Press, Cambridge (1995)

10. Gerbrandy, J.: Bisimulations on Planet Kripke. Ph. D. Thesis, University of Amsterdam (1999)

11. Jacobs, B.: The temporal logic of coalgebras via Galois algebras. Mathematical Structures in Computer Science 12, 875-903 (2002)

12. Jacobs, B.: Many-sorted coalgebraic modal logic: a model-theoretic study. Theoretical Informatics and Applications 35, 31-59 (2001)

13. Moss, L.S.: Coalgebraic logic. Annals of Pure and Applied Logic 96, 241-259 (1999)

14. Sadrzadeh, M.: Actions and Resources in Epistemic Logic. Ph.D. Thesis, University of Quebec at Montreal (2005), http://www.ecs.soton.ac.uk/ ms6/all.pdf

15. Sadrzadeh, M., Cîrstea, C.: Relating algebraic and coalgebraic logics of knowledge and update. In: Proceedings of the 7th conference on Logic and the Foundations of Game and Decision Theory, pp. 199-208, Liverpool (July 2006)

16. Rutten, J.J.M.M.: Universal coalgebra: a theory of systems. Theoretical Computer Science 249, 3-80 (2000) 